\title{
BMJ Open Understanding pathways to breast cancer diagnosis among women in the Western Cape Province, South Africa: a qualitative study
}

\author{
Jennifer Moodley, ${ }^{1,2}$ Lydia Cairncross, ${ }^{3}$ Thurandrie Naiker, ${ }^{4}$ Mariette Momberg ${ }^{2}$
}

To cite: Moodley J, Cairncross L, Naiker T, et al. Understanding pathways to breast cancer diagnosis among women in the Western Cape Province, South Africa: a qualitative study. BMJ Open 2016;6: e009905. doi:10.1136/ bmjopen-2015-009905

- Prepublication history for this paper is available online. To view these files please visit the journal online (http://dx.doi.org/10.1136/ bmjopen-2015-009905).

Received 6 September 2015 Revised 4 November 2015 Accepted 8 December 2015

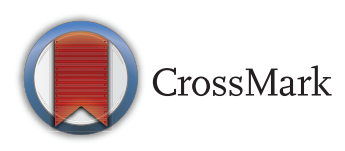

${ }^{1}$ Cancer Research Initiative, Faculty of Health Sciences, University of Cape Town, Cape Town, South Africa 'Women's Health Research Unit, Faculty of Health Sciences, School of Public Health and Family Medicine, University of Cape Town, Cape Town, South Africa ${ }^{3}$ Department of Surgery, University of Cape Town, Cape Town, South Africa ${ }^{4}$ Department of Radiation Oncology, University of Cape Town, Cape Town, South Africa

Correspondence to Professor Jennifer Moodley; jennifer.moodley@uct.ac.za

\section{ABSTRACT}

Objectives: The aim of this study was to explore and understand women's pathways to breast cancer diagnosis and factors influencing this journey.

Design and setting: Indepth interviews were conducted with clients at a tertiary level breast cancer clinic in Cape Town, South Africa. A thematic analysis was performed underpinned by the theoretical concepts of the Model of Pathways to Treatment framework.

Participants: 20 women were interviewed within 1 week of being diagnosed with breast cancer.

Results: The average time between discovery of bodily changes to breast cancer diagnosis was 8.5 months. Deficits in breast self-awareness and knowledge of breast cancer symptoms delayed women's interpretation of bodily changes as being abnormal. All women first noticed breast lumps; however, many did not perceive it as abnormal until additional symptoms were present. General good health, attribution of symptoms to ageing, and past benign breast disease resulted in women being complacent about bodily changes. Disclosure to family members served as a trigger to seek healthcare. The initial type of primary level care services women accessed was influenced by perceptions of care each service provided, finances, structural factors, and personal safety related to the physical location of services.

Conclusions: Symptom appraisal and interpretation contributed significantly to delayed presentation. To improve timely diagnosis of breast cancer, interventions that increase women's confidence in detecting breast changes, improve knowledge of breast cancer symptoms, address myths, and encourage prompt help-seeking behaviour are required.

\section{INTRODUCTION}

Breast cancer is a major public health burden and remains the commonest form of cancer among women worldwide, with 1.7 million new cases diagnosed each year. ${ }^{1}$ Global trends show that since 2008, breast cancer incidence has increased by more than
Strengths and limitations of this study

- This is the first study exploring women's pathway to breast cancer diagnosis in South Africa using the Model of Pathways to Treatment as a theoretical framework.

- The study provides a more in-depth and nuanced understanding of women's knowledge, experiences and factors influencing action taken for breast cancer diagnosis and this is a strength of qualitative research.

- This study was conducted at one clinic in the Western Cape Province and this limits its generalisability.

- A limitation of the study is that it did not include women who did not access tertiary healthcare services.

$20 \%$ and mortality by $14 \%$. In part, the increasing breast cancer incidence can be attributed to better diagnostic capabilities. ${ }^{2}$ In 2012, the majority (53\%) of new breast cancer cases were among women living in less developed countries, ${ }^{1}{ }^{2}$ where the shift toward more affluent lifestyles, particularly those associated with dietary and reproductive risk factors, has been associated with a rising burden of cancers. Lack of early detection programmes and access to treatment place women in less developed regions at a high mortality risk.

Breast cancer is the commonest form of cancer among women in South Africa (SA), with an age-standardised incidence rate of 27 per 100000 women, ${ }^{3}$ and a major cause of cancer mortality, accounting for $16 \%$ of cancer deaths among women. ${ }^{4}$ Since 1994, SA has transformed its healthcare system to one that is comprehensive, integrated, and has a primary healthcare focus. ${ }^{5}$ Like most of the resource-constrained countries, SA does not have a national mammography screening programme. Typically, women with breast symptoms self-present to primary healthcare 
facilities and are referred to secondary or tertiary level health facilities if further diagnostic work-up is required to diagnose breast cancer. Similar to other developing countries, the majority of patients present with late stage disease $^{6-8}$ which is associated with a poorer prognosis. Appropriate recognition of breast symptoms, improved access to health facilities, and accurate investigation of symptomatic women are essential to down stage with breast cancer.

For people with potential symptoms of cancer, the pathway to cancer diagnosis is complex. Studies have shown that time to diagnosis may be influenced by several factors, including knowledge and awareness of cancer symptoms, the nature of the symptoms, perception of risk, and the health system, psychological and sociocultural barriers to healthcare. ${ }^{9-17}$ Understanding the influence of these factors on the pathway women follow to breast cancer diagnosis is vital to the development of interventions.

Theoretical frameworks provide a systematic approach to understanding health behaviour and there have been calls for greater theoretical underpinning of helpseeking research. ${ }^{18} 19$ The Model of Pathways to Treatment provides a useful research framework to explore and understand patient's journeys as it takes into account the complex and dynamic nature of helpseeking behaviour. ${ }^{19}$ The Model identifies five key events in the pathway to care viz detection of bodily changes; perceived reasons to discuss symptoms with a healthcare provider; first consultation with a healthcare provider; diagnosis and start of treatment, and four important intervals between these events: the appraisal, help seeking, diagnostic and the pretreatment intervals. Importantly, this framework can be used to identify targets for interventions to promote timely diagnosis. Using the Model of Pathways to Treatment framework, we explored patient's interpretation and understanding of breast cancer symptoms, and factors influencing their pathway to diagnosis. Little is known about the pathway that women follow in reaching a breast cancer diagnosis in SA. Initial exploration using qualitative research techniques were considered most appropriate as the methodology allows for a more subtle and detailed understanding of complex issues.

\section{METHODS}

The study was conducted at the Groote Schuur Hospital Combined Breast Clinic in Cape Town, SA. The Breast Clinic is an open access, one-stop diagnostic clinic where women may present with a letter from a primary level provider (nurse practitioner or doctor). It provides a same day clinical and cytological evaluation with immediate referral to an on-site oncologist if the breast cytology is positive for malignancy. Typically, newly diagnosed patients are reviewed by a multidisciplinary team, including surgeons, oncologists, radiologists and pathologists within 1 week of diagnosis to develop a management plan. Indepth interviews were conducted with 20 purposively selected patients with newly diagnosed breast cancer between May and July 2014. Women were interviewed within 1 week of being diagnosed with breast cancer.

Data were collected using an interview guide that explored women's journey from discovery of breast changes to presentation at the Breast Clinic. Issues explored included: personal interpretation of breast changes, understanding of breast cancer signs and symptoms; perceived risk; social and family support; triggers to seeking care; access to healthcare; use of various levels of care (including alternative care); and commonly held community beliefs. Using a calendar prompt, the time interval between discovery of bodily changes to breast cancer diagnosis was elicited. The interview guide was pilot tested with two patients with newly diagnosed breast cancer and minor modifications to the wording of some questions were made. A female Xhosa-speaking research nurse trained in qualitative research methods, who was not part of the clinical management team, approached clients to take part in the study. All study participants provided written informed consent prior to the interview process. The research nurse conducted interviews lasting between 45 and $60 \mathrm{~min}$ in the language of the respondent in a private room at the Breast Clinic. Six participants were accompanied by a family member or friend, and with the consent of the participant were present during the interviews. All interviews were digitally recorded with the permission of respondents and field notes were also taken.

All recordings and field notes were translated into English, where necessary, and transcribed. Transcripts were reviewed by JM and data collection stopped once data saturation was reached. Data were entered into QSR NVivo 10 (a qualitative computer software package). A thematic analysis was performed. Initial coding categories for analysing data were drawn from the interview guides and from the theoretical concepts of the Model of Pathways to Treatment. ${ }^{19}$ JM and MM read through all the transcripts and developed initial coding categories. MM coded the data and JM reviewed the coded data. Once all the text segments had been given basic codes, the codes were categorised into basic themes by placing similar codes together. No additional themes emerged from this process. Themes were then discussed with the clinical team members (LC and TN).

\section{RESULTS}

Twenty-two women were approached to take part in the study; one declined and another was ineligible as she had been previously diagnosed with breast cancer. The mean age of the 20 women interviewed was 52 years (range 30-74 years). Thirteen participants were married, six were single and in a stable relationship and one was widowed. All but one participant had a high school or higher education. Five participants were employed, 4 
were pensioners and the remaining 11 were unemployed. Ten women had late stage cancer (ie, stage III or IV) at the time of diagnosis. The average time between discovery of breast changes and cancer diagnosis was 256 days; with an average of 164 days between discovery of changes and presentation to the first healthcare provider, that is, the appraisal and helpseeking interval, and 92 days between assessment by the first health provider and diagnosis at the tertiary hospital breast clinic, that is, the diagnostic interval.

\section{Breast cancer knowledge and perception of risk}

Many women reported no prior knowledge about breast cancer. As one participant reported:

No, not at all so when I did find the lump which apparently I have been complaining about two years ago it was difficult for me because I had no knowledge about breast cancer. (ID10)

A few had received information from: pamphlets distributed at health facilities; television and radio campaigns; healthcare professionals, and from friends or relatives who had been diagnosed with breast cancer. Information received related to breast cancer symptoms and to a lesser extent breast self-examination. However, most were only able to vaguely report on the information received and generally felt it to be insufficient.

I did have knowledge [about breast cancer] but not the full content. (ID06)

Not really, you read the pamphlets and you actually don't understand it. (ID01)

Many women did not perceive themselves as being at risk of breast cancer.

No, it [breast cancer] didn't worry me... It was almost like it [breast cancer] won't happen to me that kind of attitude that I had. (ID11)

Some women had experience of family members having breast cancer, but this did not lead to a perception of increased risk, not did it prompt women to seek early care.

My other cousin had breast cancer...she ended up dying in 2011... I have seen the pamphlets and adverts from the television about breast cancer but I was not that interested and did not pay much attention... I have never examined myself... I really do not know anything about cancer. (ID13)

\section{Discovery and initial appraisal of body changes}

For all women, a lump was the first body change noticed. Some reported accidental discovery of a breast lump, either by themselves or a partner. Others had breast changes detected as part of a clinician visit for an unrelated problem, for example, "I was at a neurologist just for my head...she checked my breasts" (ID11).

A few women were aware of the practice of breast selfexamination, either from being informed by a health professional or through watching television. Some had discovered their lump during a breast self-examination.

They also teach us when we sit there and waiting for the family planning clinic: how to examine your breast clockwise and that and how to keep your hands on your breast and when is the best time to do it and so on. Because of the knowledge that's why I examined myself I felt inconsistency on this one (pointing at right breast) that was not here and so I went to the private doctor. (ID12)

I first noticed while self examining my breasts because I heard from the television that one of the symptoms of cancer is a lump and how to check your breasts. (ID17)

However, a few women found the discussion on breast self-examination awkward, and reported feeling uncomfortable palpating their own breasts. As one participant remarked:

I don't think it is right to feel your breasts. (ID14)

Prior experience of breast conditions caused some women to interpret their symptoms as being benign.

Another thing that made me not pay attention was because I am used to have abscesses in my breasts but then they would heal and disappear but this one didn 't go away and my breast started getting darker and I decided to go to the clinic. (ID17)

It is $6 \mathrm{~cm}$ at the moment, so I didn't take note of it because...reason being when I was in high school I also found lumps in my breast and it was breast mice [fibroadenoma], so automatically I thought it's that again, so it`s nothing to worry about. (ID10)

For many, the lack of accompanying symptoms such as pain or skin changes led to a misinterpretation of the initial symptom, false sense of reassurance and delay in seeking medical care.

I didn't pay attention to the lump because it wasn't painful when it started showing. (ID16)

I stood in the mirror and looked and I was like it's okay for one of your breasts to be bigger than the other and that's normal and I didn't see the redness or any signs that one looks for and then I thought it was not breast cancer because there weren 't any of those signs of breast cancer. (ID10)

Most women reported being in good health and therefore, were less likely to be concerned about symptoms. Others had noticed general symptoms, such as loss of appetite and weight, but attributed this to ageing. 
I really do not feel sick at all. (ID08)

But we thought with age comes losing weight and eating less. (ID09)

Women reported being fearful on discovery of initial breast changes. This caused some to deny or rationalise the seriousness of the symptoms, or hope that these would go away.

I felt like you know what, whatever... I am not going still bother about going. I think I was a little bit scared because I would rather not know, than know what it is. So if it's just painful and not affecting my health and I am not getting sick then it must be okay. (ID10)

\section{Triggers to seeking care}

A few women sought medical care immediately on discovery of the initial symptom. Most women however, monitored their initial symptoms and either persistence of symptoms, an increase in size of the lump, or experience of additional symptoms—such as pain or skin changes-were triggers to seek medical care.

I felt the little lump and I thought I am going to go, but postpone and so on. And then the end of last year I felt that the lump was bigger and then I thought I needed to go but also, you know I'll go, maybe during the recess, when we off work. In about two months or maybe three months ago I saw the dent in my breast and there was a red mark and I thought okay, I must go. (ID07)

Disclosure to family members influenced some participants to seek medical care.

Because she [mother] nagged me I came. (ID01)

In February or March the lump started growing bigger and I told my husband and he took me to the doctor. (ID06)

\section{Access to healthcare}

Most women attended public healthcare facilities for financial reasons, including low transport costs, as facilities were located close to their homes and thus they could benefit from the free public sector services. Women who sought care through the private sector did so because of convenience in terms of the prompt doctor services and the longer opening times, as well as the perceived better care and safety.

Because you don't have to queue when you visit them (private practitioner). At the clinic you have to wake up early and you might not even be seen by a doctor. (ID13)

[At public hospital there is] a problem of gangsters. (ID11)

Some believed that there was little difference between private and public doctor services. The few women who reported seeking care from a traditional healer did so in conjunction with receiving care from mainstream physicians, often after being influenced by others or if they did not perceive their symptoms to be improving. Participants who sought care from a traditional healer did so when they were visiting relatives in more rural areas in the Eastern Cape Province.

Once women sought help for the breast symptoms, many were quickly referred for appropriate assessment and management to a secondary or tertiary level health service. However, a few felt they had been misdiagnosed (in both private and public health facilities), and that this delayed final diagnosis and treatment.

I examined myself and I felt a small like a pea size lump here in my breast. So I went to the doctor and he told me that it was a gland and that's all... As time went on... I started to find that it was growing and it was growing great, so I went to the doctor again, the same surgery and there was the other doctor there a lady doctor...so she sent me to the Day Hospital. (ID12)

It was last year and she was misdiagnosed by the doctor. She went to a doctor because she felt something small in her breast and then the doctor said she might have picked up something heavy. She was given pain tablets. (ID06)

\section{Competing priorities}

There was some evidence that women delayed in seeking care due to work commitments. However for one participant disclosure to a work manager resulted in influence to promptly seek care.

\section{Community views and support mechanisms}

Many women mentioned that cancer and breast cancer, in particular, was not something that was generally discussed in the community; moreover general knowledge about breast cancer seemed to be limited in the community.

I don't want to lie, I have never heard people talking about it [breast cancer]... It [breast cancer] is something that's not talked about... (ID13)

People do not really talk about it [breast cancer]. My friends never talk about it [breast cancer]. (ID10)

A few women said that when cancer was mentioned in the community, it was usually related to the death of family member or friend.

People never talk about it at all. It only when we hear that someone has got cancer or someone has died of cancer. People do not actually talk about their views about cancer. I think that people know that cancer kills people but they do not look down upon you. Another thing we hear about it when someone has died already. (ID08) 
Most women chose to tell their immediate family and close friends of their diagnosis, and appeared to have adequate support structures in place. Participants reported that family members were an important source of emotional and practical support.

\section{DISCUSSION}

Understanding pathways to cancer diagnosis is vital to the development of effective interventions to promote timely diagnosis. ${ }^{19-21}$ To the best of our knowledge, this is the first study exploring women's pathway to breast cancer diagnosis in SA using the Model of Pathways to Treatment as a theoretical framework. Similar to other studies, an important component of delayed presentation in our study was the long appraisal and help-seeking interval. $^{91416172223}$ The first event in this interval, the detection of bodily changes, requires women to be aware of normal body morphology, have knowledge of and feel comfortable with breast self-examination, and have confidence in the ability to detect changes. Many women in our study lacked such knowledge and confidence. Further research that explores why women felt discomfort in touching their breasts is required as this is key to changing health behaviour. Women reported anxiety on discovery of breast changes and as reported elsewhere, this resulted in conflicting outcomes causing some to deny the seriousness of bodily changes and dismiss it as normal, and others to seek care promptly. ${ }^{24}$ Our study points to a clear need for targeted interventions to increase women's ability and confidence to detect breast changes. Interventions will need to take into account possible discomfort with touching oneself, reported in our and other studies, ${ }^{162526}$ and the fear of self-detection of breast cancer. ${ }^{2527} 28$

Women with low breast cancer awareness are more likely to delay in presenting to a healthcare provider. $^{13} 16172930$ In our study, a major deficit in knowledge of the symptoms of breast cancer delayed women's interpretation of body changes as being abnormal. Although the initial change reported by all women was a breast lump, many did not perceive it as abnormal until additional symptoms, such as pain and skin changes, were experienced. Pain associated with breast lumps has been shown to influence women's help-seeking behaviour in different ways. ${ }^{14} 16172324$ For some women, as in our study, the absence of pain results in a misinterpretation of the lump as benign and can contribute to delays in seeking care, while others have reported that some women regard absence of pain as sinister and this is a trigger for action.

The Common-Sense Model of Illness Self-regulation suggests that for bodily changes to be perceived as a symptom, that is, as abnormal, the changes need to exceed an inference threshold for it to result in a more complete appraisal of the symptom. ${ }^{31}$ Bodily changes below the threshold are normalised. In our study, both a sense of overall good health and attribution of more general symptoms, such as loss of weight to ageing, resulted in women being complacent about bodily changes and delayed help seeking. Women with a history of benign breast disease exhibited an optimistic bias $^{19}$ in interpreting symptoms, and for some this was reinforced by downplaying of symptoms by healthcare providers.

A family history of cancer influences beliefs about cancer curability as well as perceived risk of breast cancer. $^{23}$ Despite having a family history of breast cancer, many of our participants did not perceive themselves at increased risk, nor did it influence the interpretation of a breast lump as abnormal.

Most women reported a period of monitoring of symptoms before deciding to seek care. Decisions to seek care were sometimes not acted on immediately because of contextual factors such as work priorities and relocation. The tendency for women to put other priorities ahead of their own health has been reported. ${ }^{32-34}$ Limited research is available on how job conditions may impact on health-seeking behaviour among women with cancer symptoms. ${ }^{35}$ Employers could play an important role in helping women manage work-health-seeking conflicts. For at least one participant in our study, disclosure to a manager was an important trigger to seeking care, suggesting that occupational settings could serve as an important site for education on breast cancer symptoms; this increases the likelihood of early presentation of breast cancer.

Our study confirms the findings of others on the important role that family and community members play in encouraging timely presentation as well as endorsing ill health. ${ }^{19}$ Disclosure to family members was an important trigger to seek healthcare. Further, where detection of breast changes was made by others, usually by a close family member, women sought prompt entry to health services. In contrast to other studies ${ }^{13} 16$ women did not report being fearful of isolation or abandonment in disclosing symptoms to husbands and other close family members. Most participants reported family members had offered emotional and social support for future planned breast cancer treatment. Our findings show that limited discussions on cancer, including breast cancer, occur at a community level. Further, when discussions take place, these tend to centre on cancer as a terminal disease. Community views on the poor prognosis of cancer could have resulted in initial denial of a possible cancer diagnosis, causing some to only seek help when symptoms worsened. Findings highlight the importance of breast cancer awareness campaigns for expanding the target audience from women to all members in the community, and including messages on the improved prognosis associated with an early diagnosis.

The type of primary level care services (public clinics or private general practitioners) women accessed for initial appraisal of their symptoms was influenced by perceptions of care each provided, finances, structural 
factors such as long queues, and personal safety in terms of the physical location of services. It was difficult to ascertain whether these factors led to a delay in entry to healthcare; however, other studies have shown that such factors can hinder timely medical care. ${ }^{24} 3036$ There were some reports of possible initial misdiagnosis by public and private sector practitioners causing a delay in diagnosis. Further research to explore the need for provider training in assessment of symptoms suggestive of breast cancer is required. In our study, women reported limited use of traditional healers, but such practitioners could play a larger role in entry into healthcare in rural communities.

Our study was conducted at one clinic in the Western Cape Province which limits its generalisability. However, it does provide a more indepth and nuanced understanding of women's knowledge, experiences, and factors influencing action-a strength of qualitative research. Further quantitative studies are required to confirm findings. Although retrospective recall could have affected accurate reporting of symptoms and health-seeking behaviour, conducting interviews within 1 week of diagnosis and using a calendar prompt is likely to have minimised recall bias. However, conducting the interviews within 1 week of diagnosis could also have meant that some participants were not emotionally prepared to put their story into perspective. A limitation of the study is that it only includes women who have accessed healthcare. Awareness of breast changes and factors influencing symptom interpretation and management among women who have not accessed care needs to be explored.

\section{CONCLUSION}

Symptom appraisal and interpretation contributed significantly to delayed presentation. To improve timely diagnosis of breast cancer, interventions that increase women's confidence in detecting breast changes, improve knowledge of breast cancer symptoms among women and the general community, address notions that lead to misinterpretation of symptoms, and encourage prompt help-seeking behaviour are required.

Acknowledgements The authors thank the patients for sharing their journey to cancer diagnosis, the staff at the Breast Cancer Clinic for their support, Rosemary Jacobs for assistance in data collection and Vedantha Singh for assistance in preparation of the article.

Contributors JM initiated the study and developed the study protocol. LC and TN reviewed the protocol and provided clinical oversight throughout the project. MM and JM read through the transcripts and developed initial coding categories. MM coded, and MM and JM analysed the data. JM prepared the first draft, incorporated revisions, and prepared the final draft. All the authors reviewed drafts and approved the manuscript.

Funding This research was funded by the Cancer Association of South Africa (CANSA), and the University of Cape Town, Faculty of Health Sciences.

Competing interests None declared.

Ethics approval Ethical approval to conduct the study was obtained from the Human Research Ethics Committee, University of Cape Town.
Provenance and peer review Not commissioned; externally peer reviewed.

Data sharing statement No additional data are available.

Open Access This is an Open Access article distributed in accordance with the Creative Commons Attribution Non Commercial (CC BY-NC 4.0) license, which permits others to distribute, remix, adapt, build upon this work noncommercially, and license their derivative works on different terms, provided the original work is properly cited and the use is non-commercial. See: http:// creativecommons.org/licenses/by-nc/4.0/

\section{REFERENCES}

1. Ferlay J, Soerjomataram I, Ervik M, et al. GLOBOCAN 2012, cancer incidence and mortality worldwide. International Agency for Research on Cancer, 2013. http://globocan.iarc.fr (accessed 28 Jun 2015).

2. Torre LA, Bray F, Siegel RL, et al. Global cancer statistics, 2012. CA Cancer J Clin 2015;65:87-108.

3. National Cancer Registry. Cancer in South Africa 2009 full report 2015. http://www.nioh.ac.za (accessed 28 Jun 2015).

4. Bradshaw D, Schneider M, Norman R, et al. Mortality patterns of chronic diseases of lifestyle in South Africa. In: Steyn K, Fourie J, Temple N, eds. Chronic diseases of lifestyle in South Africa: 19952005. Cape Town: South African Medical Research Council, 2006:127-50.

5. Coovadia $\mathrm{H}$, Jewkes $\mathrm{R}$, Barron $\mathrm{P}$, et al. The health and health system of South Africa: historical roots of current public health challenges. Lancet 2009;374:817-34.

6. Vorobiof DA, Sitas F, Vorobiof G. Breast cancer incidence in South Africa. J Clin Oncol 2001;19(18 Suppl):125S-7S.

7. Elgaili EM, Abuidris DO, Rahman M, et al. Breast cancer burden in central Sudan. Int $J$ Womens Health 2010;2:77-82.

8. Dickens C, Joffe M, Jacobson J, et al. Stage at breast cancer diagnosis and distance from diagnostic hospital in a periurban setting: a South African public hospital case series of over 1,000 women. Int J Cancer 2014;135:2173-82.

9. Burgess CC, Ramirez AJ, Richards MA, et al. Who and what influences delayed presentation in breast cancer? $\mathrm{Br} J$ Cancer 1998;77:1343-8.

10. Nosarti C, Crayford T, Roberts JV, et al. Delay in presentation of symptomatic referrals to a breast clinic: patient and system factors. Br J Cancer 2000;82:742-8.

11. Rauscher GH, Ferrans CE, Kaiser K, et al. Misconceptions about breast lumps and delayed medical presentation in urban breast cancer patients. Cancer Epidemiol Biomarkers Prev 2010;19: 640-7.

12. Simon $\mathrm{AE}$, Waller $\mathrm{J}$, Robb $\mathrm{K}$, et al. Patient delay in presentation of possible cancer symptoms: the contribution of knowledge and attitudes in a population sample from the United Kingdom. Cancer Epidemiol Biomarkers Prev 2010;19:2272-7.

13. Norsa'adah B, Rampal KG, Rahmah MA, et al. Diagnosis delay of breast cancer and its associated factors in Malaysian women. BMC Cancer 2011:11:141-7.

14. Dye TD, Bogale S, Hobden C, et al. Experience of initial symptoms of breast cancer and triggers for action in Ethiopia. Int $\mathrm{J}$ Breast Cancer 2012;2012:908547.

15. Forbes L, Warburton F, Richards $M$, et al. Risk factors for delay in symptomatic presentation: a survey of cancer patients. Br J Cancer 2014;111:581-8.

16. Jones $\mathrm{CE}$, Maben J, Jack RH, et al. A systematic review of barriers to early presentation and diagnosis with breast cancer among black women. BMJ Open 2014;4:e004076.

17. Jones CE, Maben J, Lucas G, et al. Barriers to early diagnosis of symptomatic breast cancer: a qualitative study of black African, black Caribbean and white British women living in the UK. BMJ Open 2015:5:e006944.

18. Scott S, Walter F. Studying help-seeking for symptoms: the challenges of methods and models. Soc Personal Psychol Compass 2010:4:531-47.

19. Scott S, Walter F, Webster A, et al. The Model of Pathways to Treatment: conceptualization and integration with existing theory. $\mathrm{Br}$ $J$ Health Psychol 2013;18:45-65.

20. Andersen RS, Vedsted P, Olesen F, et al. Patient delay in cancer studies: a discussion of methods and measures. BMC Health Serv Res 2009;9:189-93.

21. Walter F, Webster A, Scott S, et al. The Andersen Model of Total Patient Delay: a systematic review of its application in cancer diagnosis. J Health Serv Res Policy 2012;17:110-18. 
22. de Nooijer J, Lechner L, de Vries H. A qualitative study on detecting cancer symptoms and seeking medical help; an application of Andersen's Model of Total Patient Delay. Patient Educ Couns 2001;42:145-57.

23. Khakbazan Z, Taghipour A, Latifnejad Roudsari R, et al. Help seeking behavior of women with self-discovered breast cancer symptoms: a meta-ethnographic synthesis of patient delay. PLoS ONE 2014;9:e110262.

24. Unger-Saldaña K, Infante-Castañeda CB. Breast cancer delay: a grounded model of help-seeking behaviour. Soc Sci Med 2011;72:1096-104

25. Naghibi SA, Shojaizadeh D, Montazeri A, et al. Sociocultural factors associated with breast self-examination among Iranian women. Acta Med Iran 2015;53:62-8.

26. Yang RJ, Huang LH, Hsieh YS, et al. Motivations and reasons for women attending a breast self-examination training program: a qualitative study. BMC Womens Health 2010;10:23.

27. Consedine NS, Magai C, Krivoshekova YS, et al. Fear, anxiety, worry, and breast cancer screening behavior: a critical review. Cancer Epidemiol Biomarkers Prev 2004;13:501-10.

28. Al-Naggar RA, Al-Naggar DH, Bobryshev YV, et al. Practice and barriers toward breast self-examination among young Malaysian women. Asian Pac J Cancer Prev 2011;12:1173-8.

29. Ramirez A, Westcombe A, Burgess C, et al. Factors predicting delayed presentation of symptomatic breast cancer: a systematic review. Lancet 1999;353:1127-31.
30. Taib NA, Yip C, Low W. Recognising symptoms of breast cancer as a reason for delayed presentation in Asian women-the psycho-socio-cultural model for breast symptom appraisal: opportunities for intervention. Asian Pac J Cancer Prev 2011;12:1601-8.

31. Leventhal H, Brissette I, Leventhal EA. The Common-Sense Model of self-regulation of health and illness. In: Cameron LD, Leventhal $\mathrm{H}$, eds. The self-regulation of health and illness behaviour. New York: Routledge, 2003:41-65.

32. Burgess $\mathrm{C}$, Hunter MS, Ramirez AJ. A qualitative study of delay among women reporting symptoms of breast cancer. Br J Gen Pract 2001;51:967-71.

33. Majaj L, Nassar M, De Allegri M. "It's not easy to acknowledge that I'm ill': a qualitative investigation into the health seeking behavior of rural Palestinian women. BMC Womens Health 2013;13:26.

34. Taha H, Al-Qutob R, Nyström L, et al. "Voices of fear and safety" women's ambivalence towards breast cancer and breast health: a qualitative study from Jordan. BMC Womens Health 2012; 12:21.

35. Vanderpool RC, Swanberg JE, Chambers MD. A narrative review of the confluence of breast cancer and low-wage employment and its impact on receipt of guideline-recommended treatment. Glob Adv Health Med 2013;2:75-85.

36. Lam WW, Tsuchiya M, Chan M, et al. Help-seeking patterns in Chinese women with symptoms of breast disease: a qualitative study. J Public Health (Oxf) 2009;31:59-68. 